Supporting Information

\title{
Ultrafine PdCu Nanoclusters by Ultrasonic-Assisted Reduction on the LDHs/rGO Hybrid with Significantly Enhanced Heck Reactivity
}

Jin Li, Ying Song, Yanna Wang, and Hui Zhang*

State Key Laboratory of Chemical Resource Engineering, Beijing University of Chemical Technology, P.O. Box 98, Beijing 100029, China.

${ }^{*}$ Corresponding author.

Tel: +8610-6442 5872

Fax: +8610-6442 5385

E-mail: huizhang67@gst21.com; zhanghui@mail.buct.edu.cn 


\section{Experimental}

\section{Synthesis of LDHs/rGO hybrid support}

The graphite oxide (GO) colloid was synthesized via the modified Hummers method. The hierarchical nanosheet array-like hybrid support $\mathrm{Co}-\mathrm{Al}$ layered double hydroxides (LDHs)/reduced graphene oxide (rGO) was obtained by an aqueous-phase co-precipitation method developed by our lab ${ }^{2,3}$. Briefly, $50 \mathrm{mg}$ of GO colloid (5.79 $\mathrm{mg} / \mathrm{mL}$ ) was ultrasonically dispersed in $100 \mathrm{~mL}$ water for $25 \mathrm{~min}$ to give an exfoliated GO suspension at room temperature. Next, $0.025 \mathrm{~g}$ of citric acid (CA) was added into the above suspension with ultrasonication for another $5 \mathrm{~min}$ to get CA-modified GO suspension. Then, $200 \mathrm{~mL}$ of alkaline solution containing $\mathrm{NaOH}(64 \mathrm{mmol})$ and $\mathrm{Na}_{2} \mathrm{CO}_{3}(20 \mathrm{mmol})$ was added dropwise into the above CA-modified $\mathrm{GO}$ suspension until $\mathrm{pH} \sim 10$ and kept for 10 min. Subsequently, the above alkaline solution and another $100 \mathrm{~mL}$ of salt solution containing $\mathrm{Co}\left(\mathrm{NO}_{3}\right)_{2} \cdot 6 \mathrm{H}_{2} \mathrm{O}(15 \mathrm{mmol})$ and $\mathrm{Al}\left(\mathrm{NO}_{3}\right)_{3} \cdot 9 \mathrm{H}_{2} \mathrm{O}(5 \mathrm{mmol})$ were simultaneously added dropwise into the above suspension under vigorous stirring with keeping $\mathrm{pH} 10 \pm 0.1$. Finally, the resultant was aged at $65{ }^{\circ} \mathrm{C}$ for $4 \mathrm{~h}$, then centrifuged and washed with water for several times, and followed by freeze-drying to obtain hierarchical nanosheets array-like LDHs/rGO hybrid support. The pure LDHs support was synthesized by similar method without $\mathrm{GO}$ and $\mathrm{CA}$. 


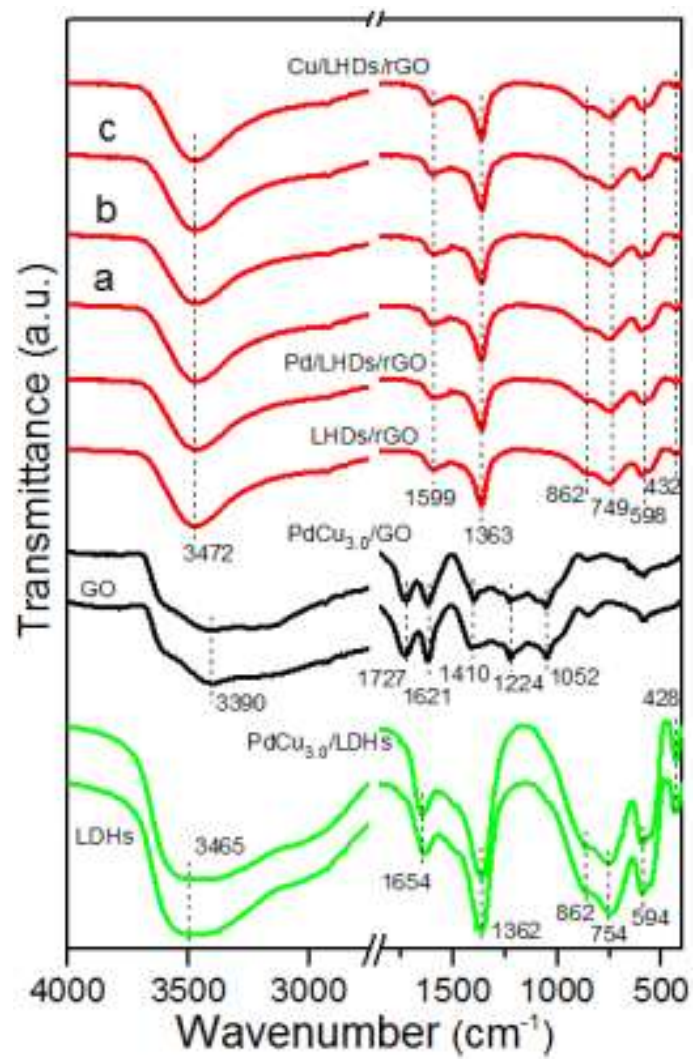

Figure S1 FTIR spectra of the $0.85-\mathrm{PdCu}_{1.5} / \mathrm{LDHs} / \mathrm{rGO}$ (a), $0.83-\mathrm{PdCu}_{3.0} / \mathrm{LDHs} / \mathrm{rGO}$ (b), 0.80-PdCu $5.5 / \mathrm{LDHs} / \mathrm{rGO}$ (c) compared with $\mathrm{Pd} / \mathrm{LDHs} / \mathrm{rGO}, \mathrm{Cu} / \mathrm{LDHs} / \mathrm{rGO}$ and related samples.

Figure S1 shows the FTIR spectra of as-synthesized $0.85-\mathrm{PdCu}_{1.5} / \mathrm{LDHs} / \mathrm{rGO}$ (a), $0.83-\mathrm{PdCu}_{3.0} / \mathrm{LDHs} / \mathrm{rGO} \quad$ (b), $\quad 0.80-\mathrm{PdCu}_{5.5} / \mathrm{LDHs} / \mathrm{rGO} \quad$ (c) compared with monometallic $\mathrm{Pd} / \mathrm{LDHs} / \mathrm{rGO}, \mathrm{Cu} / \mathrm{LDHs} / \mathrm{rGO}$ and related samples. The GO shows clearly IR bands at 1727, 1621, 1410, 1224, $1052 \mathrm{~cm}^{-1}$, assigned to the stretching vibrations of $\mathrm{C}=\mathrm{O}$ (carboxylic acid), $\mathrm{C}=\mathrm{C} / \mathrm{C}-\mathrm{C}$ (carbon skeleton), $\mathrm{C}-\mathrm{OH}$ (carboxyl), $\mathrm{C}-\mathrm{O}-\mathrm{C}$ (epoxy) and $\mathrm{C}-\mathrm{O}$ (alkoxy) ${ }^{4,5}$, respectively. However, the LDHs/rGO hybrid shows no absorption peaks corresponding to above-mentioned O-containing functions compared to GO, indicating the effective reduction of GO to rGO. Also, it shows a broad peak at $\sim 3472 \mathrm{~cm}^{-1}$ assigned to the $v(\mathrm{OH})$, one sharp peak at $\sim 1363 \mathrm{~cm}^{-1}$ and one weak peak at $862 \mathrm{~cm}^{-1}$ assigned to the $v_{3}$ (symmetrical stretching) and $v_{2}$ (out-of-plane deformation) vibrations of $\mathrm{CO}_{3}{ }^{2-}$ ions in $\mathrm{LDHs}$ phase, respectively, and the bands at $\sim 749,598$ and $432 \mathrm{~cm}^{-1}$ due to the vibrations of $\mathrm{M}-\mathrm{O}$ and $\mathrm{M}-\mathrm{OH}$ in LDHs lattice. ${ }^{2,5,6}$ Interestingly, the peak of skeletal bending vibration of $\mathrm{C}=\mathrm{C}$ at $\sim 1599 \mathrm{~cm}^{-1}$ shows an obvious red-shift compared to GO $\left(1621 \mathrm{~cm}^{-1}\right)$, implying to the possible existence of the strong GO-LDHs interaction. For series of $\mathrm{PdCu}$ alloy $\mathrm{NC}$ catalysts, the similar characteristic to corresponding support can be observed, implying considerably stable structural property after loading PdCu NCs, in line with XRD and Raman data. 

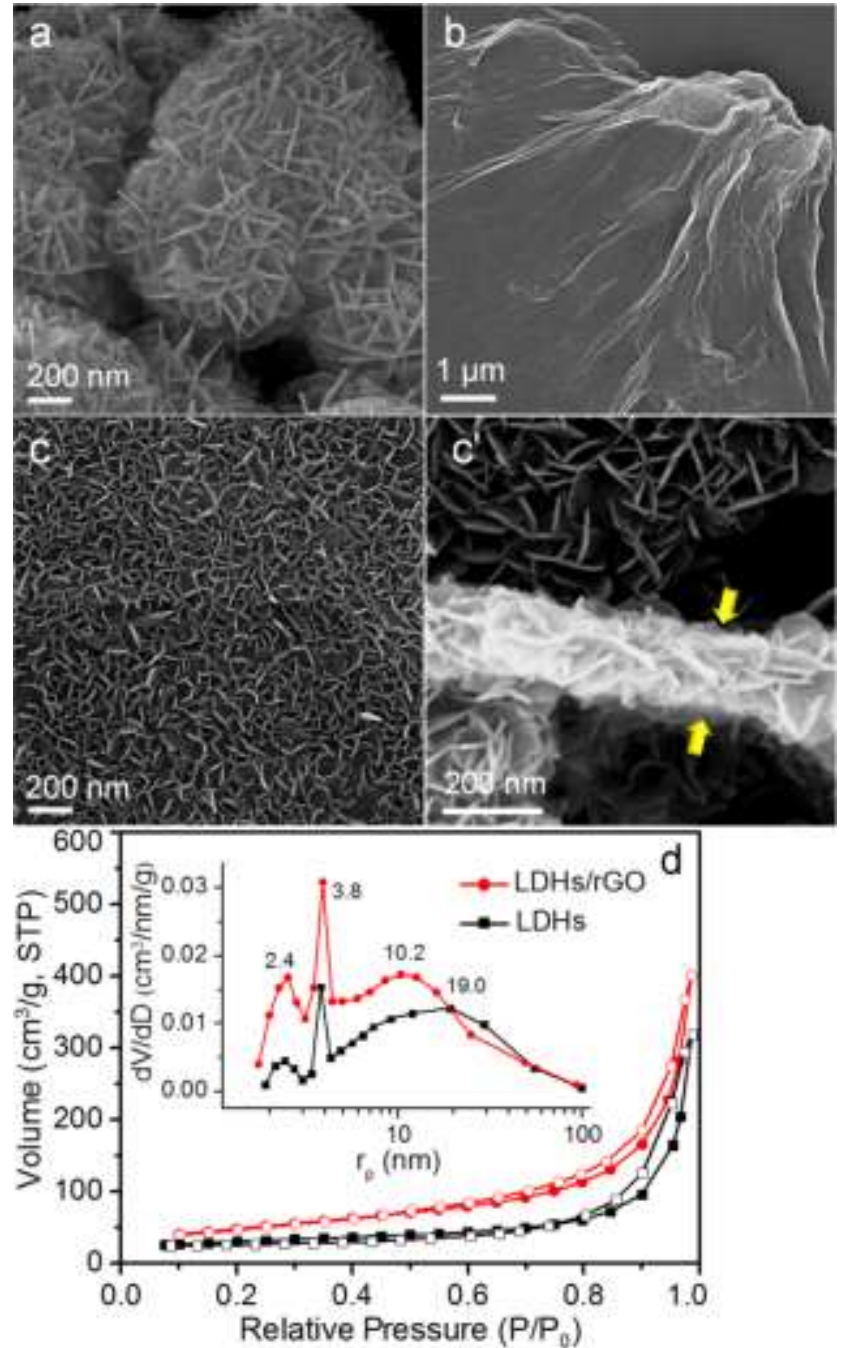

Figure S2 SEM images of the LDHs (a), GO (b) and LDHs/rGO (c and c'), and $\mathrm{N}_{2}$ adsorption-desorption isotherms (d) of LDHs/rGO and LDHs (inset: pore size distribution).

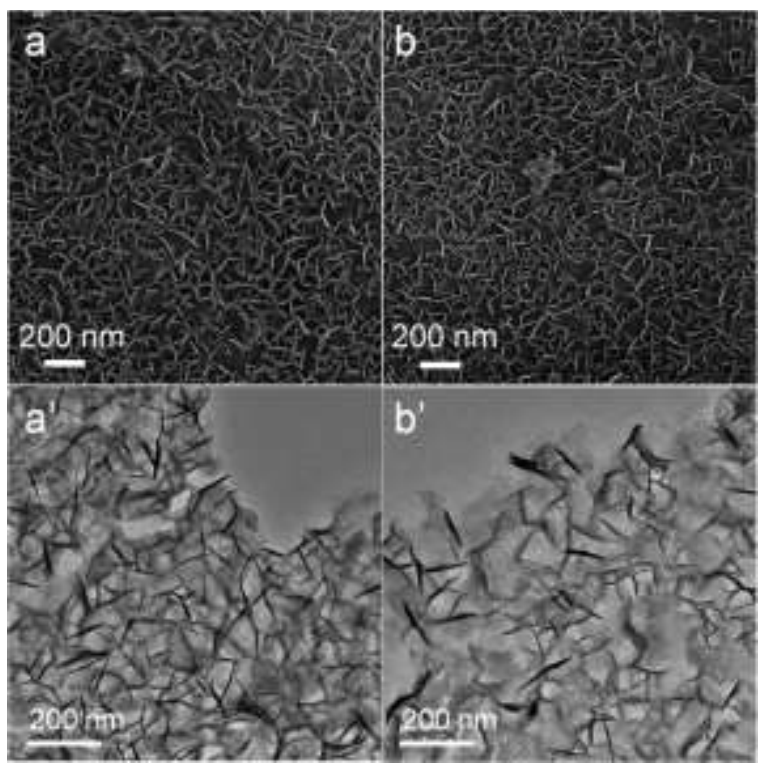

Figure S3 SEM and TEM images of the $0.85-\mathrm{PdCu}_{1.5} / \mathrm{LDHs} / \mathrm{rGO} \quad\left(\mathrm{a}, \mathrm{a}^{\prime}\right)$ and $0.80-\mathrm{PdCu}_{5.5} / \mathrm{LDHs} / \mathrm{rGO}$ (b, b'). 


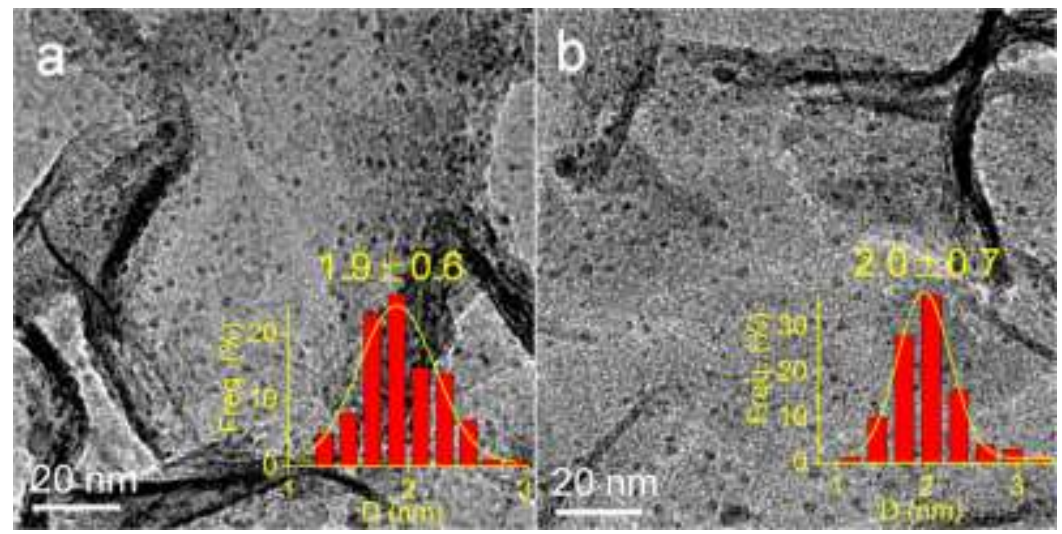

Figure S4 HRTEM images of the Pd/LDHs/rGO (a) and Cu/LDHs/rGO (b) (inset: histograms of particle size distribution).

\section{References}

(1) Cote, L. J.; Kim, F.; Huang, J. X. Langmuir-Blodgett Assembly of Graphene Oxide Single Layers. J. Am. Chem. Soc. 2009, 131, 1043-1049.

(2) Dou, L. G.; Zhang, H. Facile Assembly of Nanosheets Array-Like CuMgAl-Layered Double Hydroxide/rGO Nanohybrids for Highly Efficient Reduction of 4-Nitrophenol. J. Mater. Chem. A 2016, 4, 18990-19002.

(3) Dou, L. G.; Fan, T.; Zhang, H. A Novel 3D Oxide Nanosheet Array Catalyst Derived from Hierarchical Structured Array-Like CoMgAl-LDH/Graphene Nanohybrid for Highly Efficient $\mathrm{NO}_{\mathrm{x}}$ Capture and Catalytic Soot Combustion. Catal. Sci. Technol. 2015, 5, 5153-5167.

(4) Stankovich, S.; Dikin, D. A.; Piner, R. D.; Kohlhaas, K. A.; Kleinhammes, A.; Jia, Y. Y.; Wu, Y.; Nguyen, S. T.; Ruoff, R. S. Synthesis of Graphene-Based Nanosheets via Chemical Reduction of Exfoliated Graphite Oxide. Carbon 2007, 45, 1558-1565.

(5) Wang, Y. N.; Dou, L. G.; Zhang, H. Nanosheet Array-Like Palladium-Catalysts $\mathrm{Pd}_{\mathrm{x}} / \mathrm{rGO} @ \mathrm{CoAl}-\mathrm{LDH}$ via Lattice Atomic-Confined in situ Reduction for Highly Efficient Heck Coupling Reaction. ACS Appl. Mater. Interfaces 2017, 9, 38784-38795.

(6) Huang, S.; Zhu, G. N.; Zhang, C.; Tjiu, W. W.; Xia, Y. Y.; Liu, T. X. Immobilization of $\mathrm{Co}-\mathrm{Al}$ Layered Double Hydroxides on Grapheme Oxide Nanosheets: Growth Mechanism and Supercapacitor Studies. ACS Appl. Mater. Interfaces 2012, 4, 2242-2249. 


\section{${ }^{1} \mathrm{H}$ and ${ }^{13} \mathrm{C}$ NMR spectra of the Heck reaction products}

(1) (E)-1,2-diphenylethene: Product 1a (Table 2, entries 1, 8 and 10 ) was prepared according to the general procedure and purified by column chromatography to give a white solid.

${ }^{1} \mathrm{H}-\mathrm{NMR}\left(600 \mathrm{M}, \mathrm{CDCl}_{3}\right) ; \delta(\mathrm{ppm})$ : 7.52-7.50 (d, 4H), 7.37-7.33 (t, 4H), 7.27-7.23 (t, 2H), 7.11 (s, $2 \mathrm{H}) ;{ }^{13} \mathrm{C}-\mathrm{NMR}\left(600 \mathrm{M}, \mathrm{CDCl}_{3}\right) ; \delta$ (ppm): 137.36, 128.72, 128.68, 127.66, 126.55 .

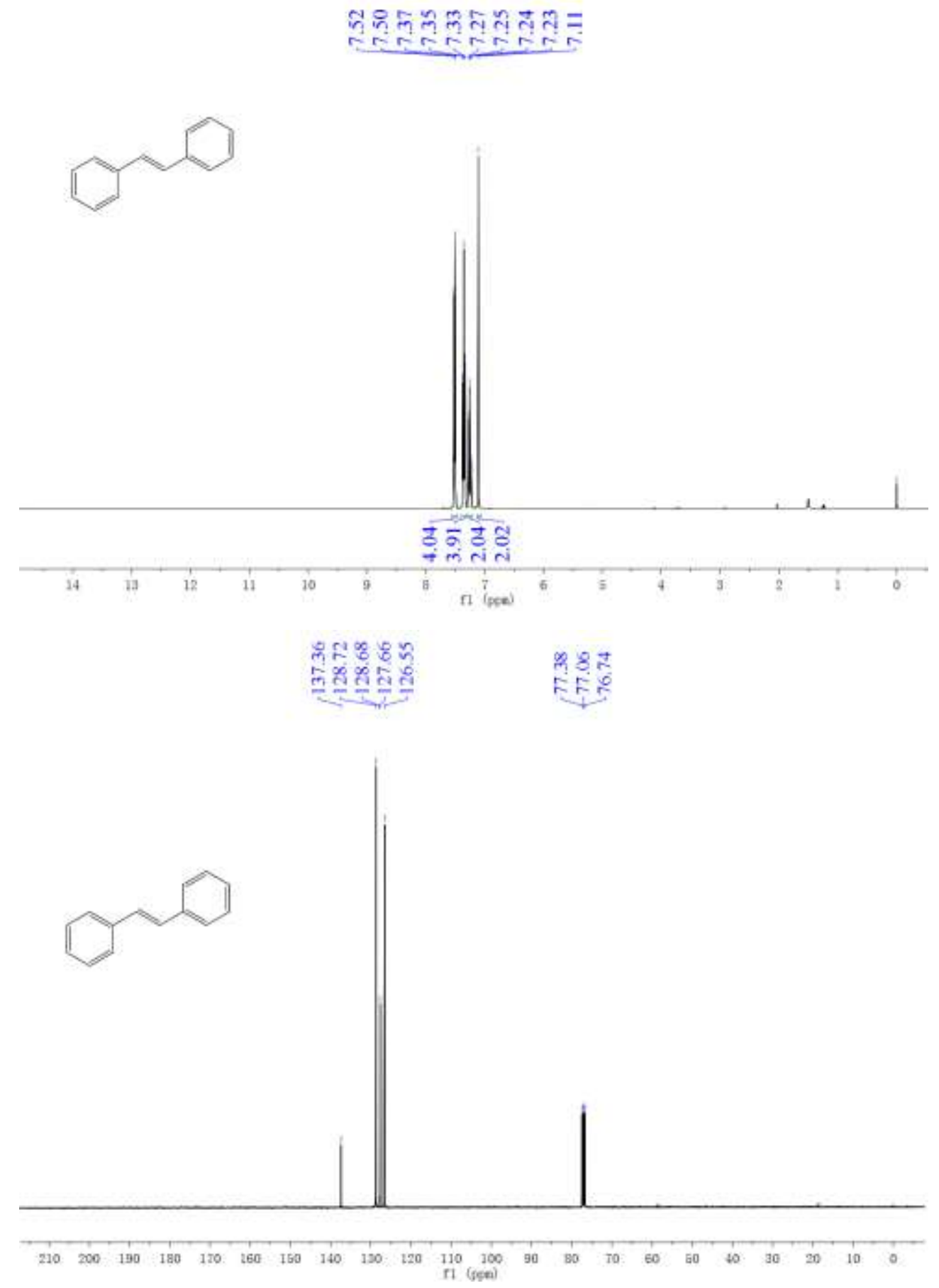

Figure S5 ${ }^{1} \mathrm{H}-\mathrm{NMR}$ (upper) and ${ }^{13} \mathrm{C}-\mathrm{NMR}$ (bottom) spectra of (E)-1,2-diphenylethene. 
(2) (E)-1-nitro-4-styrylbenzene: Product $\mathbf{1 b}$ (Table 2, entry 2) was prepared according to the general procedure and purified by column chromatography to give a yellow solid.

${ }^{1} \mathrm{H}-\mathrm{NMR}\left(600 \mathrm{M}, \mathrm{CDCl}_{3}\right) ; \delta(\mathrm{ppm}): 8.22-8.20(\mathrm{~d}, 2 \mathrm{H}), 7.70-7.65(\mathrm{~d}, 2 \mathrm{H}), 7.56-7.54(\mathrm{~d}, 2 \mathrm{H})$, 7.42-7.39 (t, 2H), 7.36-7.34 (t, 1H), 7.28-7.27 (d, 1H), 7.14-7.13 (d, 1H); ${ }^{13} \mathrm{C}-\mathrm{NMR}(600 \mathrm{M}$, $\left.\mathrm{CDCl}_{3}\right) ; \delta(\mathrm{ppm}): 146.76,143.86,136.18,133.32,128.90,128.87,127.04,126.86,126.29,124.19$.

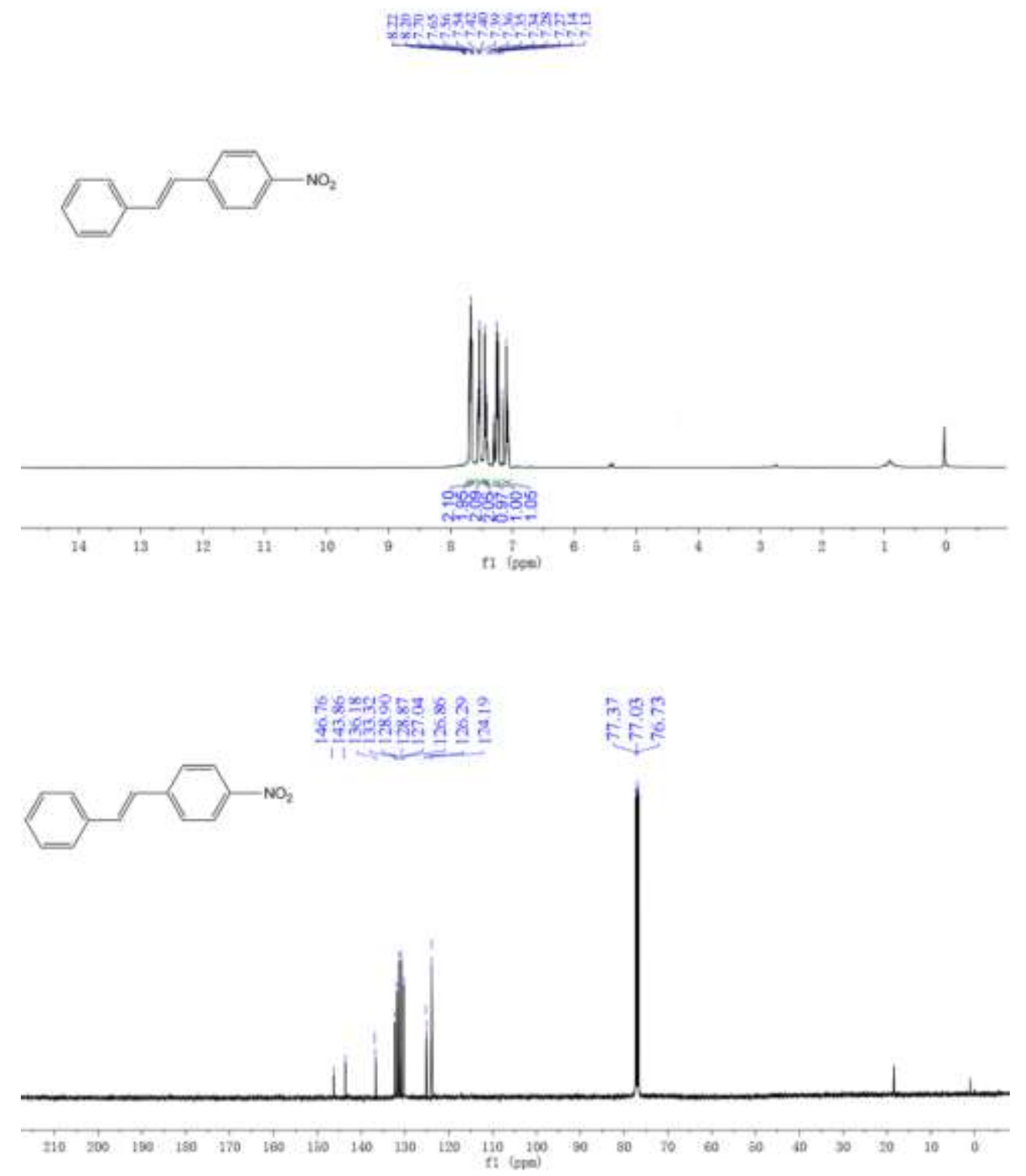

Figure S6 ${ }^{1} \mathrm{H}-\mathrm{NMR}$ (upper) and ${ }^{13} \mathrm{C}-\mathrm{NMR}$ (bottom) spectra of (E)-1-nitro-4-styrylbenzene. 
(3) (E)-1-(4-styrylphenyl) ethanone: Product 1c (Table 2, entry 3) was prepared according to the general procedure and purified by column chromatography to give a light yellow solid.

${ }^{1} \mathrm{H}-\mathrm{NMR}\left(600 \mathrm{M}, \mathrm{CDCl}_{3}\right) ; \delta$ (ppm): 7.97-7.95 (d, 2H), 7.58-7.57 (d, 2H), 7.53-7.52 (d, 2H), 7.40-7.36 (t, 2H), 7.29-7.25 (t, 1H), 7.22-7.20 (d, 1H), $7.12(\mathrm{~d}, 1 \mathrm{H}), 2.64(\mathrm{~s}, 3 \mathrm{H}) ;{ }^{13} \mathrm{C}-\mathrm{NMR}$ (600M, $\left.\mathrm{CDCl}_{3}\right) ; \delta(\mathrm{ppm}): 197.6,142.1,136.7,136.1,131.5,128.9,128.8,128.4,127.6,126.8$, $126.5,26.8$.

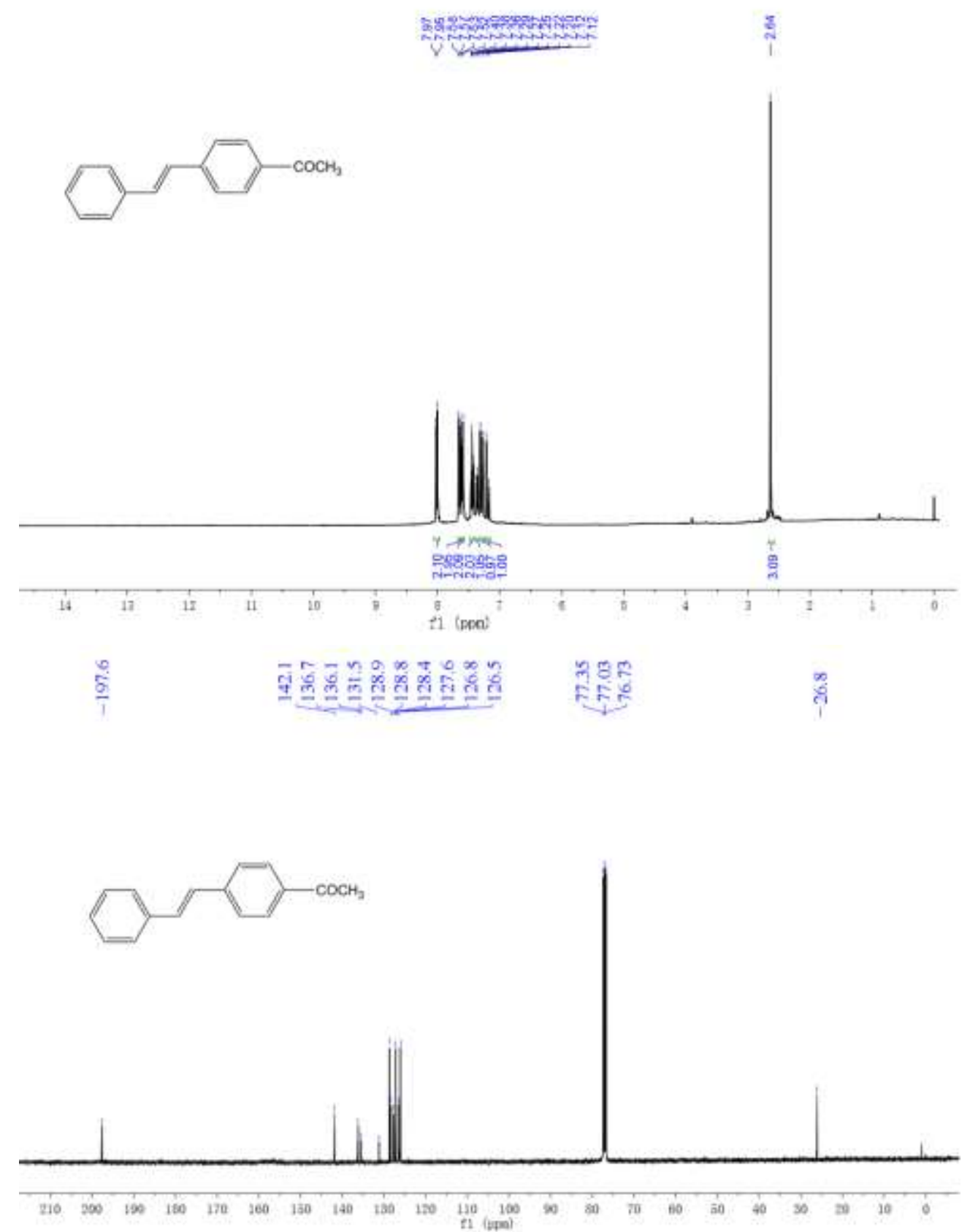

Figure S7 ${ }^{1} \mathrm{H}-\mathrm{NMR}$ (upper) and ${ }^{13} \mathrm{C}-\mathrm{NMR}$ (bottom) spectra of (E)-1-(4-styrylphenyl) ethanone. 
(4) (E)-1-methyl-4-styrylbenzene: Product 1d (Table 2, entry 4) was prepared according to the general procedure and purified by column chromatography to give a white solid.

${ }^{1} \mathrm{H}-\mathrm{NMR}\left(600 \mathrm{M}, \mathrm{CDCl}_{3}\right) ; \delta(\mathrm{ppm}): 7.55-7.54(\mathrm{~d}, 2 \mathrm{H}), 7.47-7.45(\mathrm{~d}, 2 \mathrm{H}), 7.41-7.37(\mathrm{t}, 2 \mathrm{H})$, 7.30-7.27 (t, $1 \mathrm{H}), 7.22-7.20(\mathrm{~d}, 2 \mathrm{H}), 7.07(\mathrm{~s}, 2 \mathrm{H}), 2.40(\mathrm{~s}, 3 \mathrm{H}) ;{ }^{13} \mathrm{C}-\mathrm{NMR}\left(600 \mathrm{M}, \mathrm{CDCl}_{3}\right) ; \delta(\mathrm{ppm})$ : $136.49,133.51,128.37,127.82,127.62,126.66,126.37,125.39,125.36,20.23$.

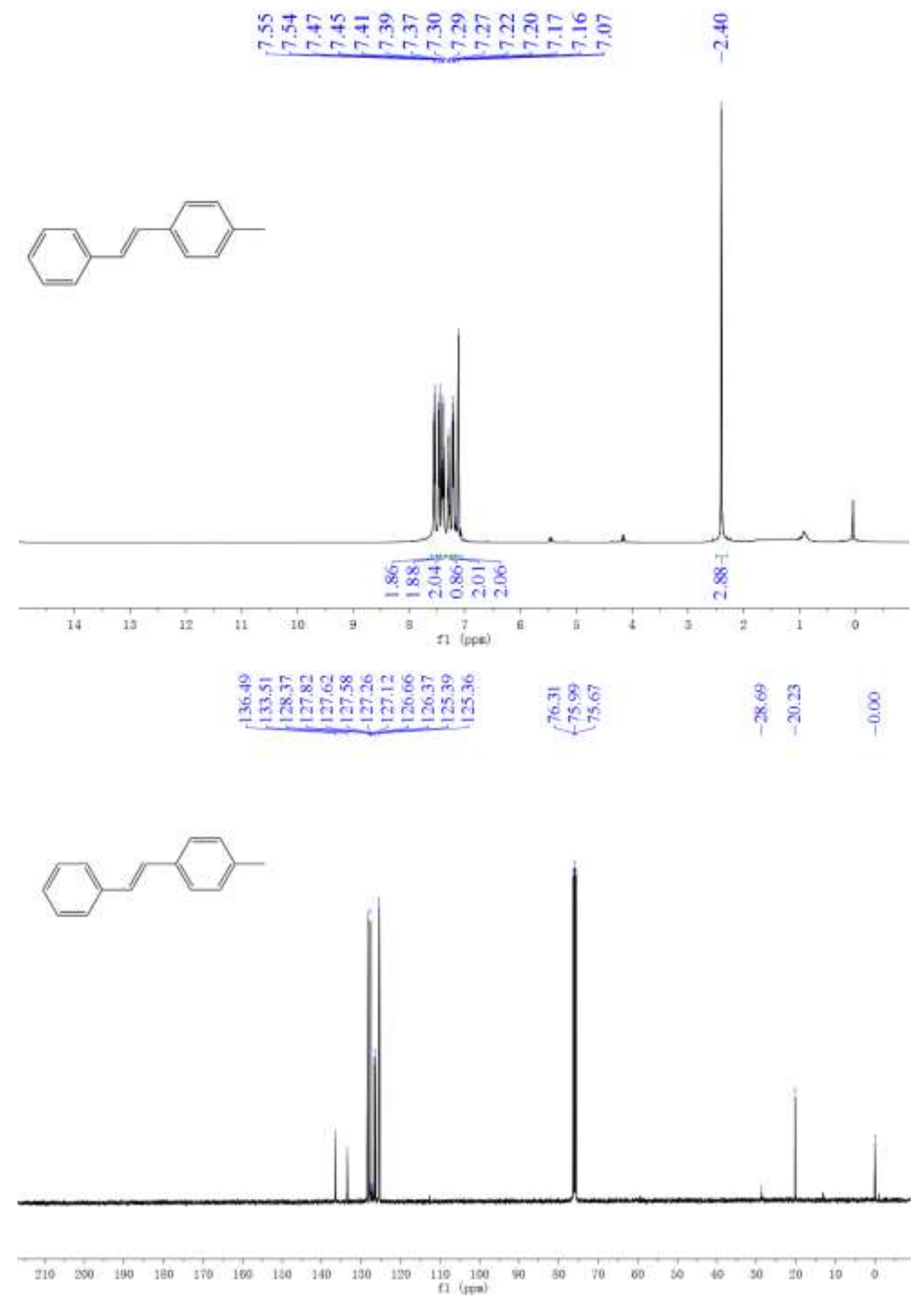

Figure S8 ${ }^{1} \mathrm{H}-\mathrm{NMR}$ (upper) and ${ }^{13} \mathrm{C}-\mathrm{NMR}$ (bottom) spectra of (E)-1-methyl-4-styrylbenzene. 
(5) (E)-1-methoxy-4-styrylbenzene: Product 1e (Table 2, entry 5) was prepared according to the general procedure and purified by column chromatography to give a white solid.

${ }^{1} \mathrm{H}$ NMR (600 MHz, $\mathrm{CDCl}_{3}$ ); $\delta$ (ppm): 7.53-7.48 (m, 4H), 7.40-7.36 (t, 2H), 7.29-7.25 (t, 1H), 7.12-7.08 (d, 1H), 7.03-6.99 (d, 1H), 6.94-6.92 (d, 2H), $3.86(\mathrm{~s}, 3 \mathrm{H}) ;{ }^{13} \mathrm{C}$ NMR (600 MHz, $\left.\mathrm{CDCl}_{3}\right)$; $\delta$ (ppm): 159.31, 137.66, 128.65, 128.22, 127.73, 127.22, 126.63, 126.26, 114.15, 55.35.

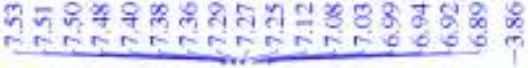

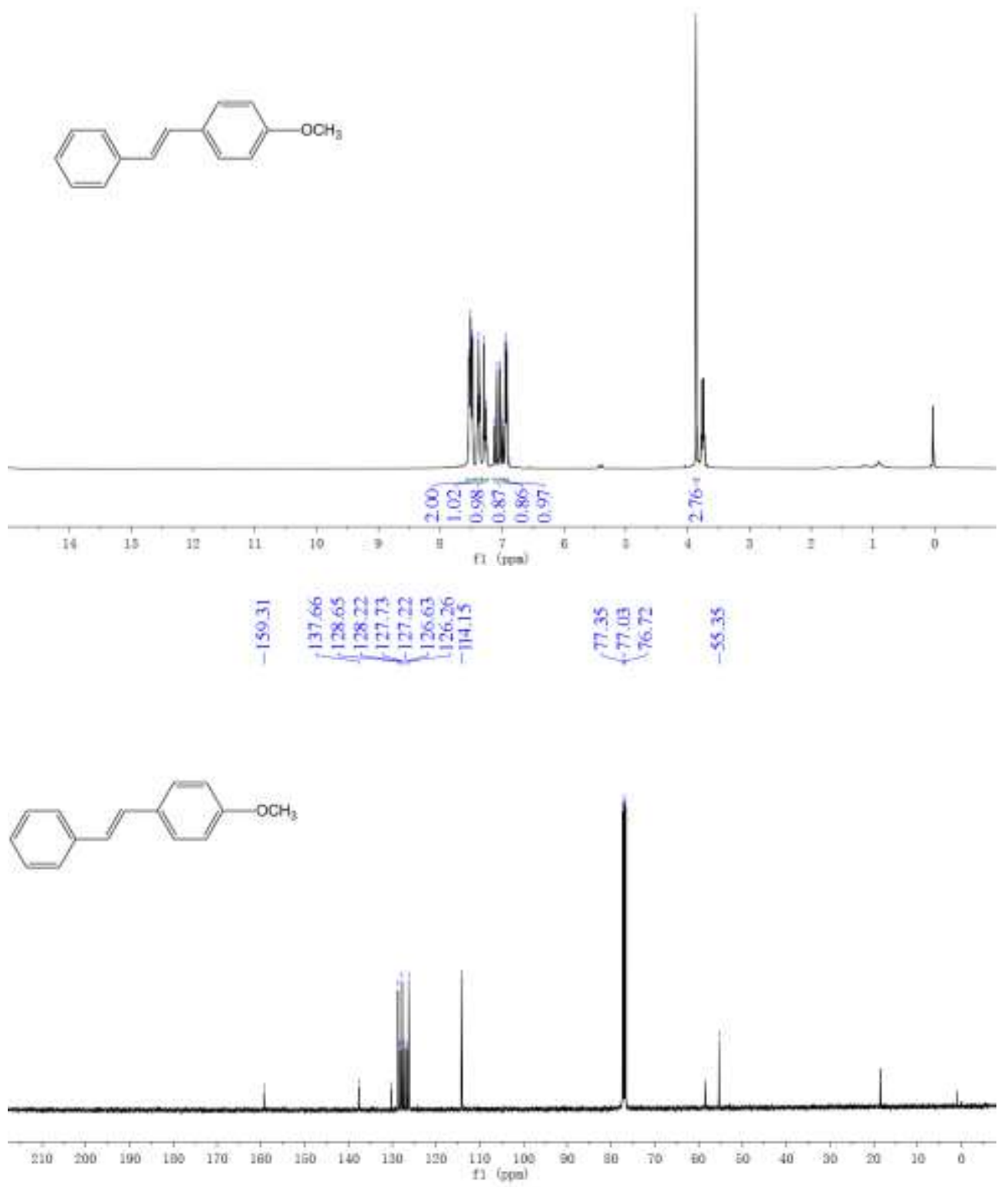

Figure S9 ${ }^{1} \mathrm{H}-\mathrm{NMR}$ (upper) and ${ }^{13} \mathrm{C}$-NMR (bottom) spectra of (E)-1-methoxy-4-styrylbenzene. 
(6) (E)-methyl cinnamate: Product $1 \mathbf{f}$ (Table 2, entries 6 and 9) was prepared according to the general procedure and purified by column chromatography to give a white solid.

${ }^{1} \mathrm{H}$ NMR (600 MHz, $\mathrm{CDCl}_{3}$ ); $\delta(\mathrm{ppm})$ : 7.75-7.71 (d, 1H), 7.56-7.54 (m, 2H), 7.44-7.40 (m, 3H), 6.49-6.45 (d, $1 \mathrm{H}), 3.84(\mathrm{~s}, 3 \mathrm{H}) ;{ }^{13} \mathrm{C}$ NMR $\left(\mathrm{CDCl}_{3}\right) ; \delta(\mathrm{ppm}): 167.45,144.89,134.40,130.32$, $128.91,128.09,117.81,51.72$.

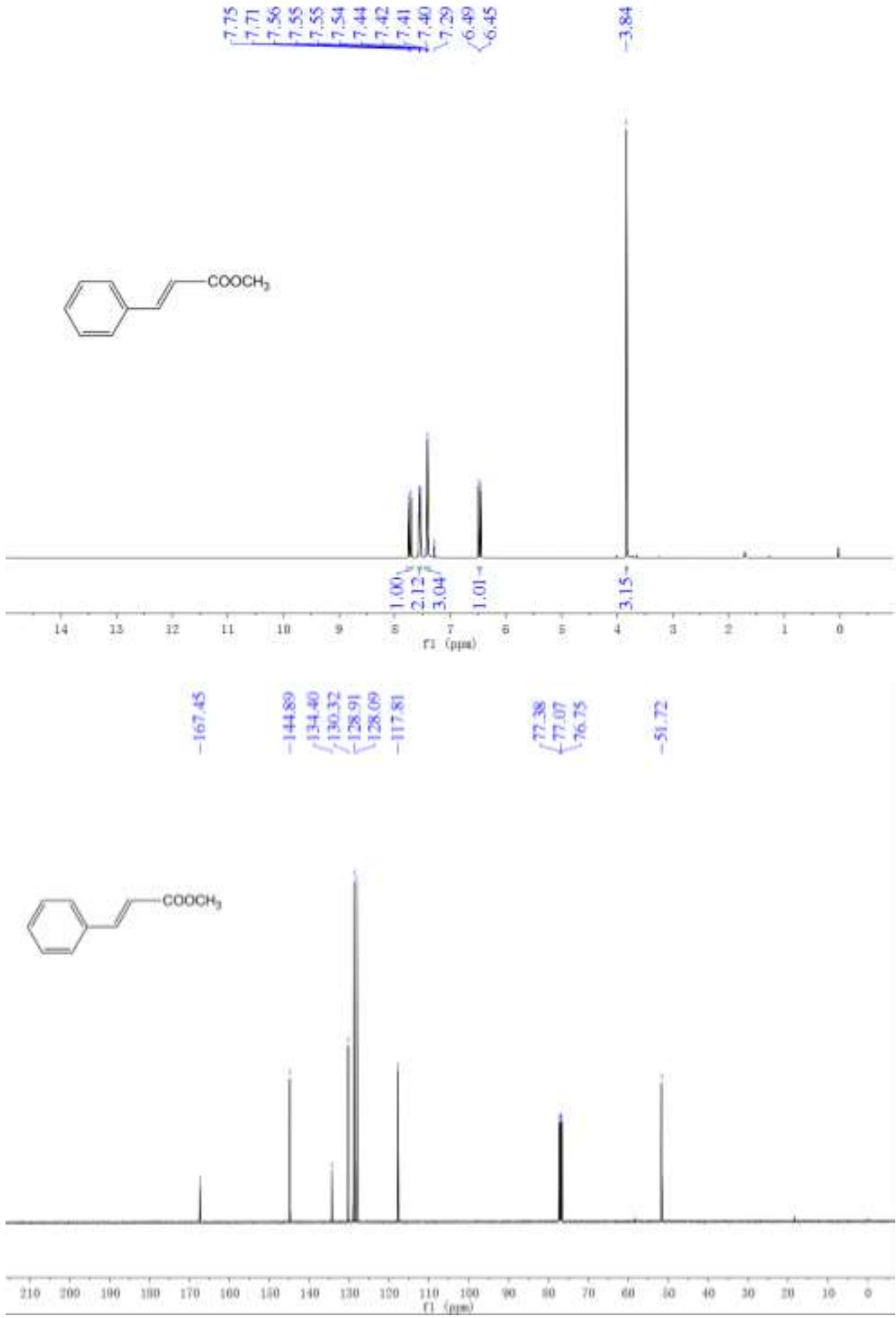

Figure S10 ${ }^{1} \mathrm{H}-\mathrm{NMR}$ (upper) and ${ }^{13} \mathrm{C}-\mathrm{NMR}$ (bottom) spectra of (E)-methyl cinnamate. 
(7) (E)-ethyl cinnamate: Product $\mathbf{1 g}$ (Table 2, entry 7) was prepared according to the general procedure and purified by column chromatography to give a white solid.

${ }^{1} \mathrm{H}$ NMR (600 MHz, $\mathrm{CDCl}_{3}$ ); $\delta(\mathrm{ppm})$ : 7.74-7.70 (d, 1H), 7.56-7.42 (m, 2H), 7.41-7.29 (m, 2H), 6.49-6.45 (d, 1H), 4.32-4.27 (m, 2H), 1.39-1.35 (t, 3H); ${ }^{13} \mathrm{C} \mathrm{NMR}\left(600 \mathrm{MHz}, \mathrm{CDCl}_{3}\right) ; \delta(\mathrm{ppm})$ : $167.03,144.60,134.48,130.23,128.89,128.06,118.29,60.52,14.35$.
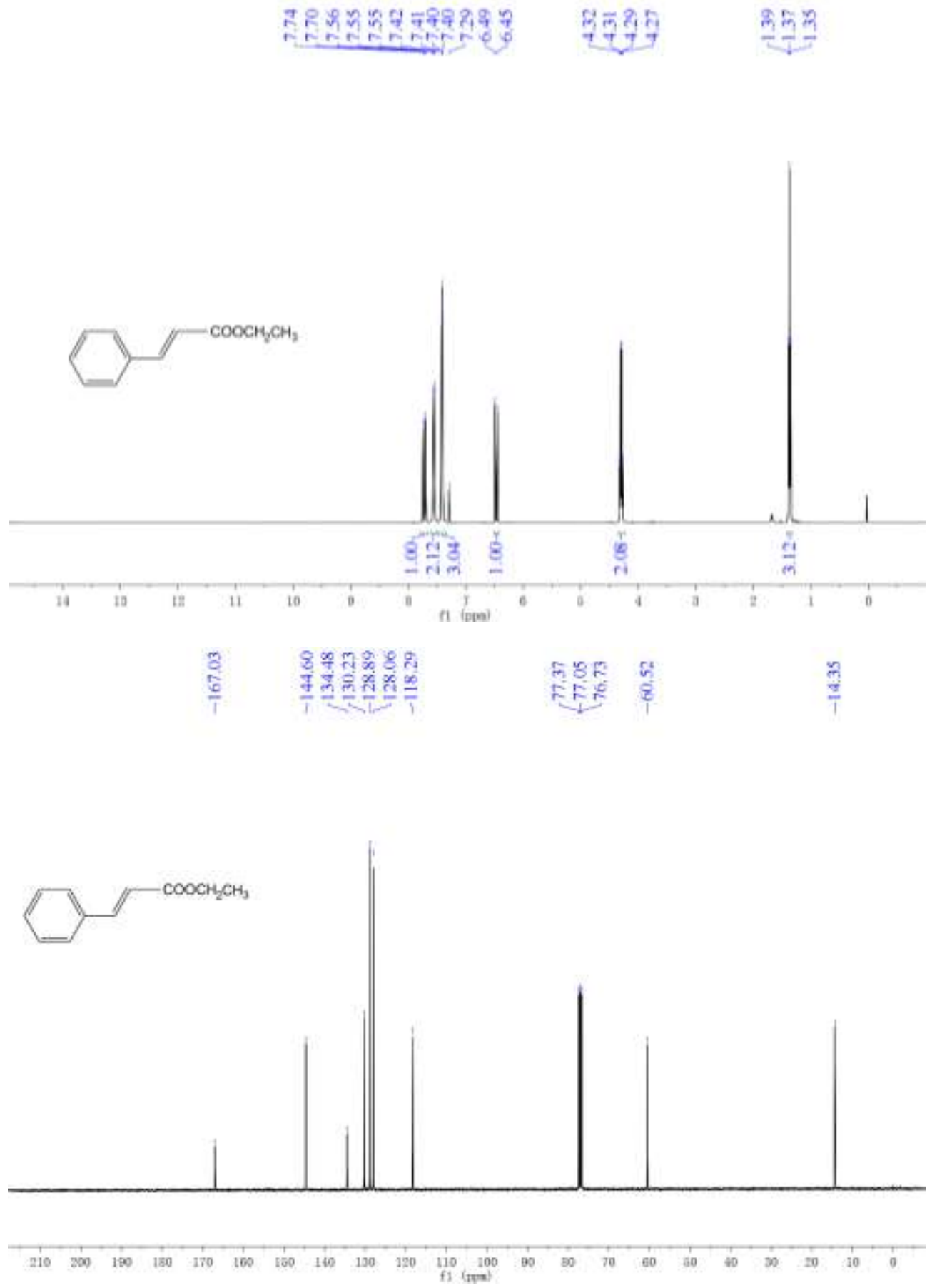

Figure S11 ${ }^{1} \mathrm{H}-\mathrm{NMR}$ (upper) and ${ }^{13} \mathrm{C}-\mathrm{NMR}$ (bottom) spectra of (E)-ethyl cinnamate. 

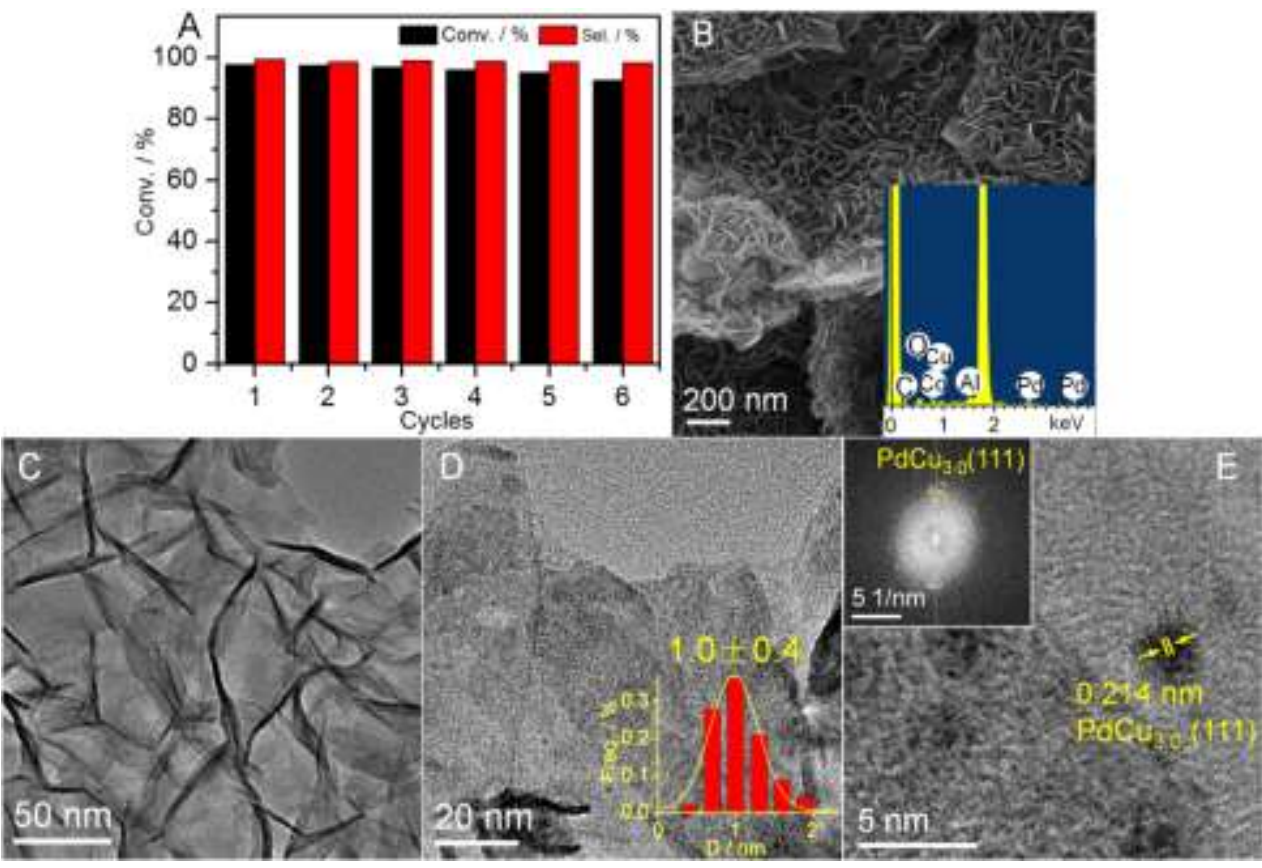

Figure S12 Recycling experiments (A) of the Heck reaction over the $0.01-\mathrm{PdCu}_{3.0} / \mathrm{LDHs} / \mathrm{rGO}$, and SEM (B) and HRTEM images (C-E) of the recycled catalyst after 6 runs (insets: EDX spectra, histogram of particle size distribution and FFT).

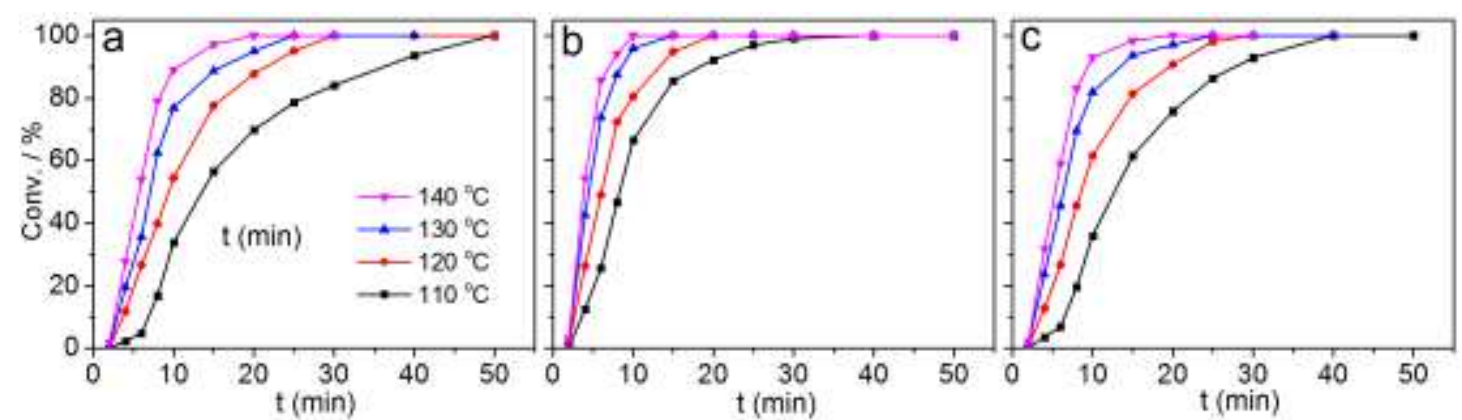

Figure S13 Conv. - time $(t)$ plots for the Heck reaction of PhI with styrene over the $0.85-\mathrm{PdCu}_{1.5} / \mathrm{LDHs} / \mathrm{rGO}$ (a), $0.83-\mathrm{PdCu}_{3.0 /} \mathrm{LDHs} / \mathrm{rGO}$ (b) and $0.80-\mathrm{PdCu}_{5.5} / \mathrm{LDHs} / \mathrm{rGO}$ (c) at various reaction temperatures. 


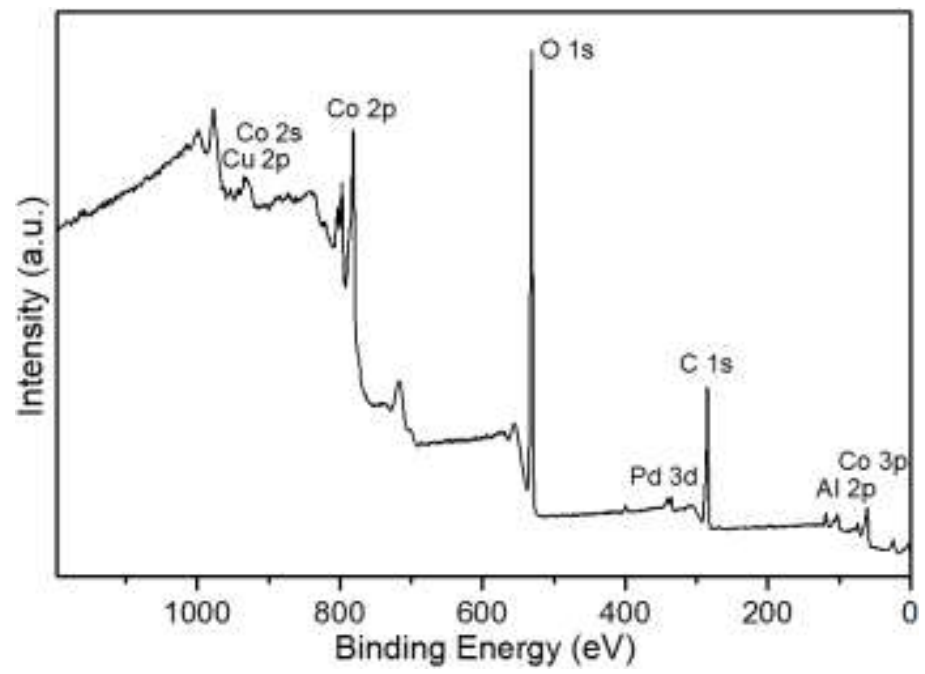

Figure S14 XPS survey spectra of the $0.83-\mathrm{PdCu}_{3.0} / \mathrm{LDHs} / \mathrm{rGO}$.
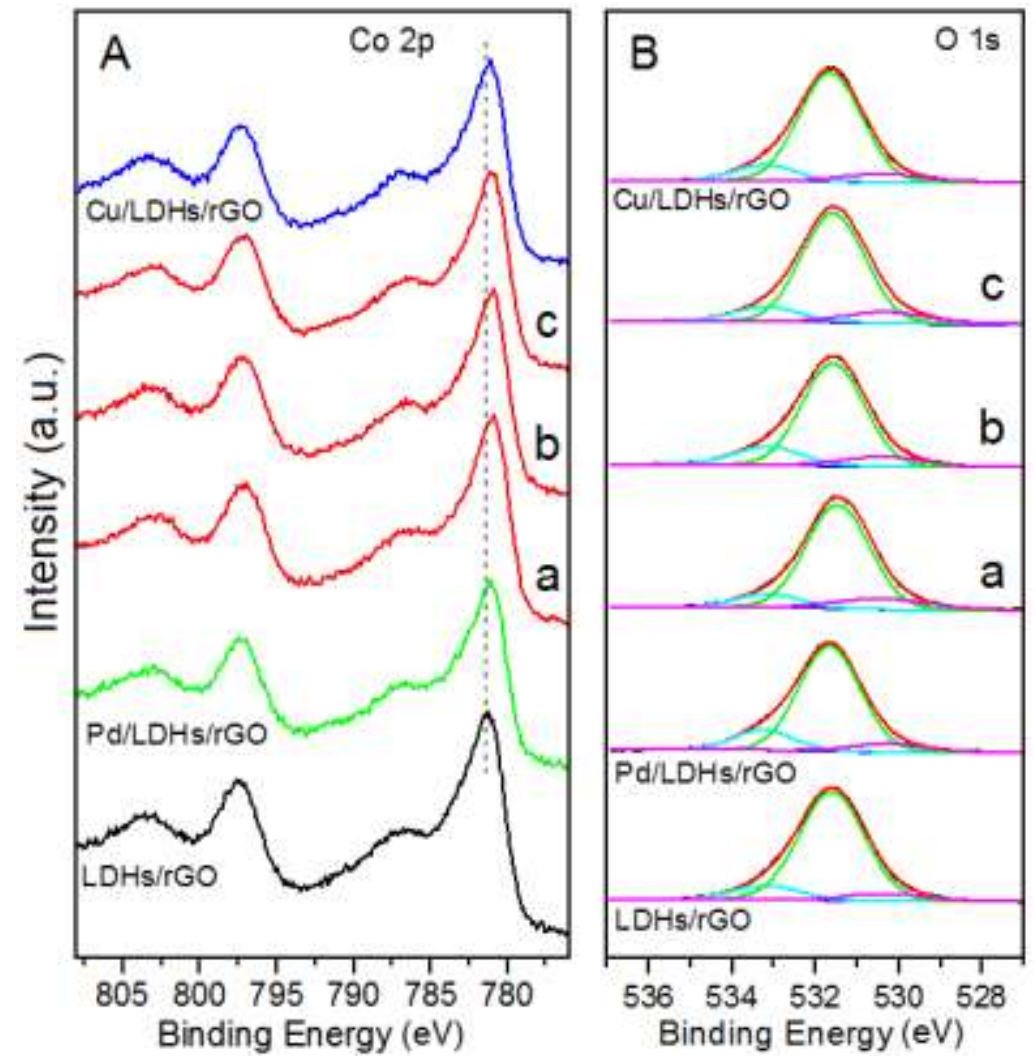

Figure S15 Co $2 \mathrm{p}$ (A) and $\mathrm{O} 1 \mathrm{~s}$ (B) XPS spectra of the $0.85-\mathrm{PdCu}_{1.5} / \mathrm{LDHs} / \mathrm{rGO}$ (a) $0.83-\mathrm{PdCu}_{3.0} / \mathrm{LDHs} / \mathrm{rGO}$ (b) and $0.80-\mathrm{PdCu}_{5.5} / \mathrm{LDHs} / \mathrm{rGO}$ (c) compared with $\mathrm{Pd} / \mathrm{LDHs} / \mathrm{rGO}$, $\mathrm{Cu} / \mathrm{LDH} / \mathrm{rGO}$ and $\mathrm{LDHs} / \mathrm{rGO}$. 


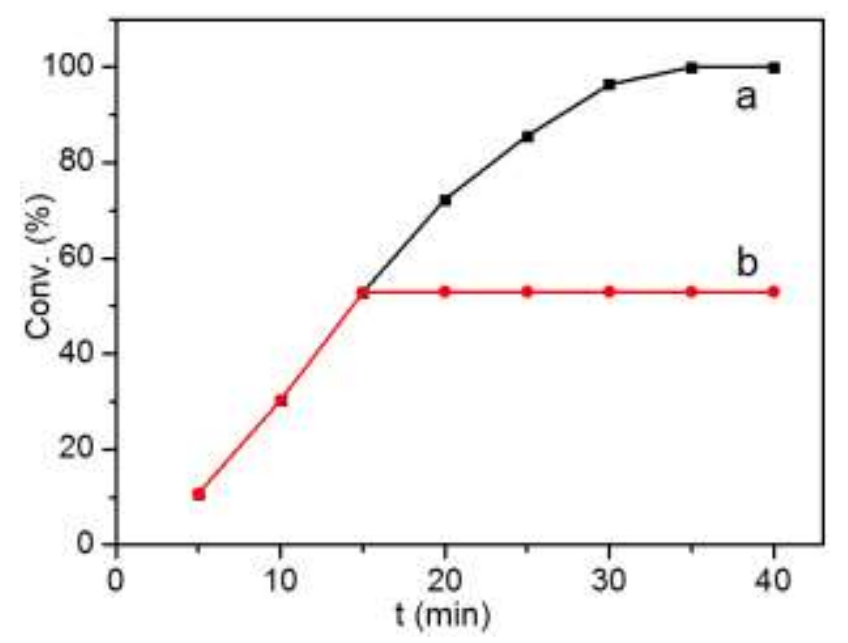

Figure S16 Conv. $-t$ plots for the Heck reaction of $\mathrm{PhI}$ with styrene over the 0.01-PdCu $3.0 / \mathrm{LDHs} / \mathrm{rGO}$ (a) and with filtrate (b).

Table S1. XRD parameters of the catalysts $m-\mathrm{PdCu}_{\mathrm{x}} / \mathrm{LDHs} / \mathrm{rGO}$ compared with related samples.

\begin{tabular}{cccccccc}
\hline Samples & $d_{003} / \mathrm{nm}$ & $d_{110} / \mathrm{nm}$ & $\mathrm{a} / \mathrm{nm}^{a}$ & $\mathrm{c} / \mathrm{nm}^{a}$ & $\begin{array}{c}D_{003} / \\
\mathrm{nm}^{b}\end{array}$ & $\begin{array}{c}D_{110} / \\
\mathrm{nm}^{b}\end{array}$ & $\mathrm{I}_{110} / \mathrm{I}_{003}$ \\
\hline Cu/LDHs/rGO & 0.7757 & 0.1536 & 0.3072 & 2.327 & 10.0 & 22.8 & 0.30 \\
$0.85-\mathrm{PdCu}_{1.5} / \mathrm{LDHs} / \mathrm{rGO}$ & 0.7757 & 0.1537 & 0.3074 & 2.327 & 10.0 & 22.8 & 0.30 \\
$0.83-\mathrm{PdCu}_{3.0} / \mathrm{LDHs} / \mathrm{rGO}$ & 0.7618 & 0.1539 & 0.3078 & 2.285 & 10.0 & 22.8 & 0.29 \\
$0.80-\mathrm{PdCu} 5.5 / \mathrm{LDHs} / \mathrm{rGO}$ & 0.7688 & 0.1539 & 0.3078 & 2.306 & 10.1 & 22.9 & 0.31 \\
$\mathrm{Pd} / \mathrm{LDHs} / \mathrm{rGO}$ & 0.7751 & 0.1536 & 0.3072 & 2.325 & 10.0 & 21.2 & 0.29 \\
$\mathrm{LDHs} / \mathrm{rGO}$ & 0.7670 & 0.1537 & 0.3074 & 2.301 & 9.90 & 18.9 & 0.29 \\
$\mathrm{PdCu}_{3.0} / \mathrm{LDHs}$ & 0.7635 & 0.1539 & 0.3078 & 2.291 & 12.6 & 29.1 & 0.19 \\
$\mathrm{LDHs}$ & 0.7648 & 0.1535 & 0.3070 & 2.294 & 12.7 & 28.6 & 0.19 \\
$\mathrm{PdCu}_{3.0} / \mathrm{GO}$ & - & 0.7914 & 1.5827 & - & - & 12.0 & - \\
$\mathrm{GO}$ & - & 0.7949 & 1.5898 & - & - & 12.4 & - \\
\hline
\end{tabular}

${ }^{a}$ Based on hexagonal crystal system, $\mathrm{a}=2 d_{110}, \mathrm{c}=3 d_{003}$.

${ }^{b}$ Calculated by Scherrer formula, $D_{\mathrm{hkl}}=0.89 \lambda /(\beta \cos \theta)(\lambda$ is the X-ray wavelength $(0.1542 \mathrm{~nm}), \beta$ is haft-height width in radian, $\theta$ is Bragg diffraction angle in degree). 
Table S2. Kinetic results of the Heck reaction over the $m-\mathrm{PdCu} / \mathrm{LDHs} / \mathrm{rGO}{ }^{a}$

\begin{tabular}{|c|c|c|c|c|c|}
\hline Catalysts & $\mathrm{T}(\mathrm{K})$ & $k\left(\min ^{-1}\right)^{b}$ & $\overline{\mathrm{R}^{2}}$ & $\overline{E_{\mathrm{a}}(\mathrm{kJ} / \mathrm{mol})^{\mathrm{c}}}$ & $\overline{\mathrm{R}^{2}}$ \\
\hline \multirow{4}{*}{$0.85-\mathrm{PdCu}_{1.5} / \mathrm{LDHs} / \mathrm{rGO}$} & 383.15 & 0.0696 & 0.9839 & \multirow{4}{*}{60.1} & \multirow{4}{*}{0.9921} \\
\hline & 393.15 & 0.1189 & 0.9872 & & \\
\hline & 403.15 & 0.1701 & 0.9855 & & \\
\hline & 413.15 & 0.2833 & 0.9871 & & \\
\hline \multirow{4}{*}{$0.83-\mathrm{PdCu}_{3.0} / \mathrm{LDHs} / \mathrm{rGO}$} & 383.15 & 0.1669 & 0.9858 & \multirow{4}{*}{46.9} & \multirow{4}{*}{0.9947} \\
\hline & 393.15 & 0.2295 & 0.9837 & & \\
\hline & 403.15 & 0.3478 & 0.9905 & & \\
\hline & 413.15 & 0.4763 & 0.9911 & & \\
\hline \multirow{4}{*}{$0.80-\mathrm{PdCu}_{5.5} / \mathrm{LDHs} / \mathrm{rGO}$} & 383.15 & 0.0972 & 0.9756 & \multirow{4}{*}{53.6} & \multirow{4}{*}{0.9871} \\
\hline & 393.15 & 0.1356 & 0.9860 & & \\
\hline & 403.15 & 0.2076 & 0.9845 & & \\
\hline & 413.15 & 0.3289 & 0.9839 & & \\
\hline
\end{tabular}

${ }^{a}$ Reaction conditions: dosage of catalyst (Pd: $0.3 \mathrm{~mol} \%$, based on $\left.\mathrm{PhI}\right), \mathrm{PhI}(1.0 \mathrm{mmol})$, styrene $(1.2 \mathrm{mmol}), \mathrm{K}_{2} \mathrm{CO}_{3}(3.0 \mathrm{mmol})$, mixed solvent: $\mathrm{V}_{\mathrm{DMF}} / \mathrm{V}_{\mathrm{H} 2 \mathrm{O}}=12 / 4(\mathrm{~mL} / \mathrm{mL})$, and reaction temperature (T) of 383.15-413.15 K under atmospheric conditions. ${ }^{b}$ Upon the equation: $\ln \left(C_{t} / C_{0}\right)$ $=-k t\left(C_{\mathrm{t}}\right.$ is the concentration of $\mathrm{PhI}$ at $t$ time, $\mathrm{mol} / \mathrm{L} ; C_{0}$ is the initial concentration of iodobenzene, $\mathrm{mol} / \mathrm{L} ; k$ is the reaction rate constant, $\min ^{-1}$; and $t$ is the reaction time, min). ${ }^{c}$ Upon the Arrhenius equation: $k=\operatorname{Aexp}\left(-E_{\mathrm{a}} / \mathrm{RT}\right)\left(E_{\mathrm{a}}\right.$ is the apparent activation energy, $\mathrm{kJ} / \mathrm{mol} ; \mathrm{A}$ is the pre-exponential factor, $\min ^{-1} ; \mathrm{R}$ is the molar gas constant, $8.314 \mathrm{~J} \cdot \mathrm{mol}^{-1} \cdot \mathrm{K}^{-1}$, and $\mathrm{T}$ is the absolute temperature, $\mathrm{K}$ ).

Table S3. XPS data of the $m-\mathrm{PdCu}_{\mathrm{x}} / \mathrm{LDHs} / \mathrm{rGO}$ compared with related samples.

\begin{tabular}{|c|c|c|c|c|c|c|c|}
\hline \multirow{2}{*}{ Samples } & \multirow{2}{*}{$\begin{array}{c}\mathrm{Pd}^{0} 3 \mathrm{~d}_{5 / 2} \\
(\mathrm{eV})\end{array}$} & \multirow{2}{*}{$\begin{array}{l}\mathrm{Pd}^{0} /\left(\mathrm{Pd}^{\mathrm{II}}\right. \\
\left.+\mathrm{Pd}^{0}\right)(\%)\end{array}$} & \multirow{2}{*}{$\begin{array}{c}\mathrm{Cu} 2 \mathrm{p}_{3 / 2} \\
(\mathrm{eV})\end{array}$} & \multirow{2}{*}{$\begin{array}{c}\text { Co } 2 p \\
(e V)\end{array}$} & \multicolumn{3}{|c|}{$\mathrm{O} 1 \mathrm{~s}(\mathrm{eV})$} \\
\hline & & & & & $-\mathrm{OH}$ & $\mathrm{O}^{2-}$ & $\mathrm{H}_{2} \mathrm{O}$ \\
\hline $\mathrm{Pd} / \mathrm{LDHs} / \mathrm{rGO}$ & 335.40 & 84.4 & - & 781.19 & $531.67(78.6 \%)$ & 530.28 & 533.26 \\
\hline $0.85-\mathrm{PdCu}_{1.5} / \mathrm{LDHs} / \mathrm{rGO}$ & 335.15 & 88.4 & 932.62 & 781.09 & $531.47(78.3 \%)$ & 530.41 & 533.15 \\
\hline $0.83-\mathrm{PdCu}_{3.0} / \mathrm{LDHs} / \mathrm{rGO}$ & 334.96 & 92.0 & 932.83 & 780.91 & $531.58(79.3 \%)$ & 530.38 & 533.16 \\
\hline 0.80-PdCu $5.5 / \mathrm{LDHs} / \mathrm{rGO}$ & 335.11 & 89.3 & 932.69 & 780.96 & $531.58(77.7 \%)$ & 530.31 & 533.18 \\
\hline $\mathrm{Cu} / \mathrm{LDHs} / \mathrm{rGO}$ & - & - & 932.42 & 781.20 & $531.65(81.1 \%)$ & 530.39 & 533.23 \\
\hline LDHs/rGO & - & - & - & 781.36 & $531.61(82.5 \%)$ & 530.30 & 533.19 \\
\hline
\end{tabular}

